\title{
Inter-rater reliability and validity of risk of bias instrument for non-randomized studies of exposures: a study protocol
}

\author{
Maya M. Jeyaraman ${ }^{1,2^{*}}$, Nameer Al-Yousif ${ }^{1}$, Reid C. Robson ${ }^{3}$, Leslie Copstein ${ }^{1}$, Chakrapani Balijepalli ${ }^{4}$, \\ Kimberly Hofer ${ }^{5}$, Mir S. Fazeli ${ }^{5}$, Mohammed T. Ansari ${ }^{6}$, Andrea C. Tricco ${ }^{3,7,8}$, Rasheda Rabbani ${ }^{1,2}$ and \\ Ahmed M. Abou-Setta ${ }^{1,2}$
}

\begin{abstract}
Background: A new tool, "risk of bias (ROB) instrument for non-randomized studies of exposures (ROB-NRSE)," was recently developed. It is important to establish consistency in its application and interpretation across review teams. In addition, it is important to understand if specialized training and guidance will improve the reliability in the results of the assessments. Therefore, the objective of this cross-sectional study is to establish the inter-rater reliability (IRR), inter-consensus reliability (ICR), and concurrent validity of the new ROB-NRSE tool. Furthermore, as this is a relatively new tool, it is important to understand the barriers to using this tool (e.g., time to conduct assessments and reach consensus - evaluator burden).

Methods: Reviewers from four participating centers will apprise the ROB of a sample of NRSE publications using ROB-NRSE tool in two stages. For IRR and ICR, two pairs of reviewers will assess the ROB for each NRSE publication. In the first stage, reviewers will assess the ROB without any formal guidance. In the second stage, reviewers will be provided customized training and guidance. At each stage, each pair of reviewers will resolve conflicts and arrive at a consensus. To calculate the IRR and ICR, we will use Gwet's AC, statistic.

For concurrent validity, reviewers will appraise a sample of NRSE publications using both the Newcastle-Ottawa Scale (NOS) and ROB-NRSE tool. We will analyze the concordance between the two tools for similar domains and for the overall judgments using Kendall's tau coefficient.

To measure evaluator burden, we will assess the time taken to apply ROB-NRSE tool (without and with guidance), and the NOS. To assess the impact of customized training and guidance on the evaluator burden, we will use the generalized linear models. We will use Microsoft Excel and SAS 9.4, to manage and analyze study data, respectively.

Discussion: The quality of evidence from systematic reviews that include NRSE depends partly on the study-level ROB assessments. The findings of this study will contribute to an improved understanding of ROB-NRSE and how best to use it.
\end{abstract}

Keywords: Inter-rater reliability, Inter-consensus reliability, Concurrent validity, Risk of bias, ROB, NRS, NRSE, Crosssectional study, Non-randomized studies

\footnotetext{
* Correspondence: maya.jeyaraman@umanitoba.ca

${ }^{1}$ The George \& Fay Yee Center for Healthcare Innovation, University of

Manitoba, 363-753 McDermot Avenue, Winnipeg, Manitoba R3E 0T6, Canada

${ }^{2}$ Department of Community Health Sciences, University of Manitoba,

Winnipeg, Canada

Full list of author information is available at the end of the article
}

(c) The Author(s). 2020 Open Access This article is distributed under the terms of the Creative Commons Attribution 4.0 International License (http://creativecommons.org/licenses/by/4.0/), which permits unrestricted use, distribution, and reproduction in any medium, provided you give appropriate credit to the original author(s) and the source, provide a link to the Creative Commons license, and indicate if changes were made. The Creative Commons Public Domain Dedication waiver (http://creativecommons.org/publicdomain/zero/1.0/) applies to the data made available in this article, unless otherwise stated. 


\section{Background}

Systematic reviews inform healthcare decision-making by summarizing the best available evidence [1]. Critical appraisal of the available evidence via assessment of the risk of bias (ROB) in the studies included in a systematic review plays a key role in determining the strength or quality of the systematic review [2]. Although evidence from randomized controlled trials (RCTs) is generally considered superior to that of non-randomized studies (NRS) to prove causation [2], it is important to include evidence from NRS when evidence from RCTs is indirect, imprecise, inconsistent, inapplicable, or unavailable $[3,4]$. Having said that, it is important for systematic reviewers to be aware of the potential biases associated with NRS designs, and the best practices to minimize the impact of these biases on the effect estimate [5].

Many quality assessment tools are available to assess the methodological quality of non-randomized, observational studies of exposures (NRSE) [6-8] The Newcastle-Ottawa scale (NOS) is the most widely used [7] and has eight items divided into three domains (selection, comparability, and either outcome or exposure assessment for cohort and case-control studies, respectively). NOS uses a star system with a maximum of 1 star assigned to all eight items (except for one item under comparability domain, which receives a maximum of 2 stars); total scores can range between 0 to 9 stars [7]. Although the NOS is widely used, it was reported to have poor inter-rater reliability (IRR) [5].

In 2016, the Cochrane Methods Bias (CMB) group and the Cochrane Non-randomized Studies of Interventions Methods Group, along with a large team of experts, published the "Risk of Bias in Non-randomized Studies of Interventions" (ROBINS-I) [9] tool. The ROBINS-I tool guides adjudications on the risk of bias (ROB) of nonrandomized studies (NRS) of interventions (NRSI), by comparing it to a hypothetical target randomized controlled trial (RCT) that the NRSI best emulates (even if the RCT would be unethical or unfeasible) [2, 9]. As ROBINS-I was developed to assess ROB in interventional studies ("intentional exposures"), there was no clarity on its usefulness in assessing ROB in NRS of environmental, nutritional, or other exposures ("unintentional exposures") [10]. Unlike ROBINS-I, most previously available instruments neither use signaling questions nor the comparison to an ideal RCT concept [10].

In recent years, with an improved understanding of the potential effects of study design, study conduct, and study reporting, there has been a major shift from using checklists for assessing study quality (or just reporting per se), to assessing ROB $[11,12]$. Study quality refers to how a study was designed to avoid systematic errors during the conduct, analysis, and interpretation/reporting of the results and conclusions. Risk of bias refers to factors that could have systematically biased the study design, interpretation, analysis, presentation, or reporting of the outcome measures. Due to the intensified focus on the field of exposure assessment by the Grading of Recommendations, Assessment, Development and Evaluation (GRADE) Working Group and the need for harmonizing the rating of NRSI and the NRSE in the context of GRADE [10], a collaborative project led by a team of experts at the University of Bristol (UK), McMaster University (Canada), and the Environmental Protection Agency (USA) proposed to create a new instrument "Risk of Bias in Non-randomized Studies of Exposures" (ROBINS-E) [10]. ROBINS-E is currently under development, and as of the end of 2019, there were no official draft versions available for review [13]. What is currently available is a preliminary tool developed by the GRADE working group named the "ROB instrument for NRS of exposures" [10, 14]; for simplicity we will use the acronym ROB-NRSE to refer to this tool. ROB-NRSE was developed by making significant semantic and conceptual modifications to the ROBINS-I tool. In 2019, a user's guide to its application in the context of GRADE was also published [15].

The authors suggest following three steps in the evaluation of ROB using ROB instrument for NRS of exposures (ROB-NRSE) [10]:

(1) Step I: Describing the systematic review question (e.g., Population, Exposure, Comparator, Outcomes-PECO)

(2) Step II: Describing ideal target experiment, confounders and co-exposures

(3) Step III: Outcome-specific ROB evaluation of the included studies by the raters using the ROBNRSE [10]

Similar to ROBINS-I, ROB-NRSE is composed of seven domains to assess bias due to confounding, selection of participants, classification of exposures, departures from intended exposures, missing data, measurement of outcomes, and selection of reported results [9]. Each of the seven domains contain multiple signaling questions with five response options (yes, probably yes, no, probably no, or no information) to guide domain level ROB adjudications [9]. The ROB adjudications are categorized as follows: low risk, moderate risk, serious risk, critical risk, or no information.

As ROB-NRSE is the most current, publicly available version modeled after the ROBINS-I tool, we conducted this cross-sectional study to establish ample evidences on its reliability and validity in order to improve the consistency in its application and in how it is interpreted across various systematic reviews that include NRSE. Inter-rater reliability (IRR) refers to the reproducibility 
or consistency of decisions between two reviewers and is a necessary component of validity [16, 17]. Interconsensus reliability (ICR) refers to the comparison of consensus assessments across pairs of reviewers in the participating centers. Concurrent validity refers to the extent to which the results of the instrument or tool can be trusted [17]. Furthermore, it is important to understand the barriers to using this tool (e.g., time to conduct assessments and reach consensus-evaluator burden).

\section{Methods/design}

Using methods similar to those described previously for the evaluation of the ROBINS-I tool [18], an international team of experienced researchers from four participating centers will collaboratively undertake this study. The major objectives are the following:

I. Measure the IRR and ICR between reviewers when assessing ROB of NRSE using ROB-NRSE (without and with customized training and guidance)

II. Measure the concurrent validity of ROB-NRSE

III. Measure the evaluator burden (time taken to apply ROB-NRSE, time taken to arrive at a consensus, time taken to apply NOS)

In order to address the above objectives, we will conduct a cross-sectional analytical study on a sample of NRSE publications following this protocol. This protocol will be registered with the Open Science Framework (https://osf.io/). The final study manuscript will be reported according to the STROBE-cross-sectional checklist [19-21]. We plan to report any protocol amendments in the final study manuscript.

\section{I: Inter-rater reliability and inter-consensus reliability}

Our first objective is to evaluate the IRR of ROB-NRSE at first stage, without customized training and guidance document from principal investigator, and then at the second stage, with customized training and guidance. At both stages, assessors will have access to the publicly available detailed guidance [22]. For the second stage, a customized guidance document will be developed using Microsoft word (Word v1.5, Microsoft Corp., Redmond, WA, USA), by a senior member of the team holding $\mathrm{PhD}$ degree (MJ). Following review and feedback by another experienced senior member of the team (MA), we will finalize the document. The guidance document will contain simplified decision rules, additional guidance for advanced concepts, and clarifications on answering signaling questions that will guide reviewers in making adjudications for each domain in ROB-NRSE tool. Once developed, we will send the guidance document to all the reviewers, for help with adjudications in the second stage of the project. Additionally, one training session (via Skype) will be organized by a trainer (MJ), who is a senior member of the team and the developer of the customized guidance document. During the training session, the trainer will review the guidance document with all the reviewers and provide clarifications. We will use the following methods to assess IRR and ICR.

\section{Participating centers}

We will involve two pairs of reviewers (LC, NA, RCR, $\mathrm{CB}$, and $\mathrm{KH}$ ) with varied levels of experience and academic degrees attained, from multiple research teams to assess IRR and ICR. The participating teams are as follows: (coordinating center) The Knowledge Synthesis platform, George \& Fay Yee Center for Healthcare Innovation, University of Manitoba (Canada) (MJ, AMAS, LC, NA, RR); Knowledge Synthesis Team, Knowledge Translation Program, Li Ka Shing Knowledge Institute of St. Michael's Hospital, Unity Health Toronto (Canada) (ACT and RCR); Evidinno Outcomes Research Inc. (Canada) (KH and MSF); Pharmalytics Group, Vancouver (Canada) (CB).

\section{Sample size calculation}

We have calculated the sample size (number of NRSE publications) required for IRR assessments $(n=44)$ by taking into account a 5\% type I error, $80 \%$ statistical power, and an assumed error margin of 30\% [23-25]. As suggested by Gwet [23, 24], we assumed the chanceagreement probability $\left(P_{\mathrm{e}}\right)$ as zero (best-case scenario) and estimated the required sample size for IRR using the formulas and calculations available at: http://agreestat. com/blog_irr/sample_size_determination.html. We obtained the observed-agreement probability $\left(P_{\mathrm{a}}\right)$ between reviewers required for sample size calculation from an initial pilot testing of 10 NRSE publications.

\section{Sample selection}

We propose to use a convenience sample of prospective cohort publications published in English $(n=44)$, (based on the sample size calculations) identified from a recent systematic review. We will then identify one prespecified outcome (the primary outcome of each study), for ROB appraisals for each of the included NRSE. If a study does not report a primary outcome, the principal investigator will identify an important outcome reported in the study, for ROB appraisal. With the help of content experts, we will identify a list of confounders and important co-exposures for the specific association of interest reported in each of the included NRSE publications.

\section{Data collection}

After the initial pilot testing on 10 studies, we will proceed with ROB assessments for IRR. We will advise the reviewers to review the any available general 
guidelines for ROBINS-I provided by the developers of the ROBINS-I tool available at https://methods. cochrane.org/news/robins-i-tool. This would reflect normal practice for new researchers that have not had previous training/expertise with the tool. We will also advise all reviewers in the participating centers to read the full report of each included NRSE prior to making assessments. Reviewers will have the list of confounders and important co-exposures available during their assessments. At first, two reviewers will independently assess the ROB for the included NRSE using ROB-NRSE tool, without using any formal training or customized guidance. For each included NRSE, the two reviewers will assess the seven domains of ROB-NRSE tool as, low $R O B$, moderate $R O B$, serious $R O B$, critical $R O B$, or no information [9] (Table 1). At the end, the two reviewers will resolve conflicts and arrive at a consensus.

As a next step, each pair of reviewers will independently re-assess the same set of NRSE following formal training and using a customized guidance sheet following the initial "without guidance" ROB assessments. At the end of the assessments, again the reviewers will meet to resolve conflicts and arrive at a consensus. All studies are assessed first without guidance, before any withguidance assessments, to prevent the possibility of withguidance assessment influencing without-guidance assessment. The principal investigator (MJ) at the coordinating center will coordinate this process among reviewers in the different participating centers.

Upon completion, the collaborating center will collect, organize, and transfer the $\mathrm{ROB}$ assessment data from various reviewers to an Excel workbook, prior to proceeding with the data analysis. We will then assess and report the IRR and ICR for ROB assessments "without guidance" and "with guidance," separately.

\section{Data analysis}

An experienced biostatistician (RR) from the collaborating center will conduct all the analyses in collaboration with the other members of the research team. We will transfer all collected data from the Microsoft Excel workbook (Excel v14, Microsoft Corp., Redmond, WA, USA) to SAS (9.4), (SAS Institute Inc., Cary, NC, USA) for analysis. The kappa $(\kappa)$ statistic is typically used to assess IRR as it corrects for the "chance" agreement between the two reviewers and allows for different types of disagreements to have differing weights [26]. The chance-agreement probability evaluated by the $\mathrm{k}$ statistic assumes that all observed ratings may yield agreements by chance, thus leading to unpredictable results in the presence of high agreement between reviewers [27]. The $\mathrm{AC}_{1}$ statistic developed by Gwet [27] calculates the true overall chance agreement in the presence of high agreement reviewers, thus yielding values closer to "true" IRR
[28]. We will also analyze the inter-consensus reliability (ICR) using Gwet's $\mathrm{AC}_{1}$ statistic [27].

The agreements among reviewers (IRR and ICR) will be categorized as follows [29]: poor (0), slight (0.1-0.2), fair (0.21-0.4), moderate (0.41-0.6), substantial (0.61-0.8), or near perfect (0.81-0.99). We will tabulate the $\mathrm{AC}_{1}$ values and the $95 \%$ confidence intervals (CIs) separately (without or with guidance), as shown in Table 2. Additionally, we will assess the correlations between adjudications made during both the stages ("with guidance" and "without guidance") for each of the reviewer to ensure that the effect of training and guidance is not biased.

\section{II: Concurrent validity}

The second objective of this study is to evaluate the concurrent validity of the ROB instrument for NRS of exposures compared with NOS. Concurrent validity refers to how well a newly developed tool is correlated to similar domains of a widely used tool at the same point in time [30]. In other words, concurrent validity evaluates the extent to which there is concordance in judgment for similar domains in both the tools that are being compared [30]. Currently, there is no "gold standard" tool to asses ROB in NRSE. Hence, to assess the concurrent validity of the ROB instrument in NRS of exposures tool, we propose to use NOS, as it is the most commonly used quality assessment tool for NRSE that had been previously recommended by Cochrane [31].

In this cross-sectional study, we will explore the concordance between assessments made on similar domains in ROB-NRSE and NOS, and the overall assessments for each included NRSE.

\section{Data collection}

As mentioned previously, we will use a sample of NRSE $(n=44)$ for assessments of concurrent validity. We have compared and matched both NOS and the ROB instrument in NRS of exposures tool (as shown in Tables 3 and 4) to identify the items that completely overlap, partially overlap, or unique to each tool. Since the theoretical construct differs between NOS (methodological quality) and ROB-NRSE (ROB), we did not expect a complete match between all domains.

For the assessment of concurrent validity, one reviewer (MJ) with expertise in systematic reviews will assess NOS on a sample of NRSE $(n=44)$. We will then compare these NOS adjudications with the after-consensus adjudications of ROB-NRSE (done after customized training and guidance by two pairs of reviewers), for the same set of studies that were used for the ICR assessments.

We will calculate the correlation between the two tools for each of the domains and for the overall 


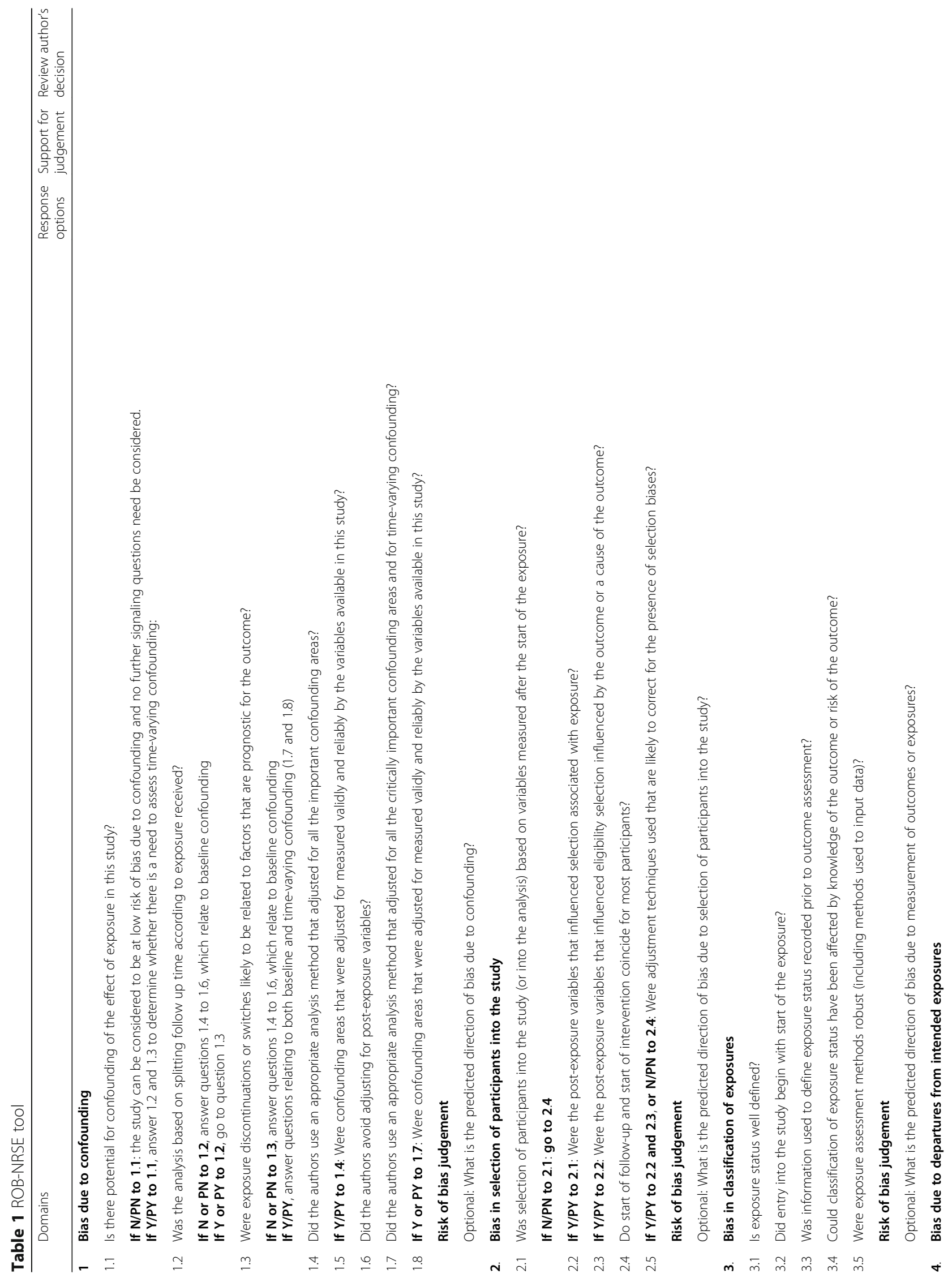




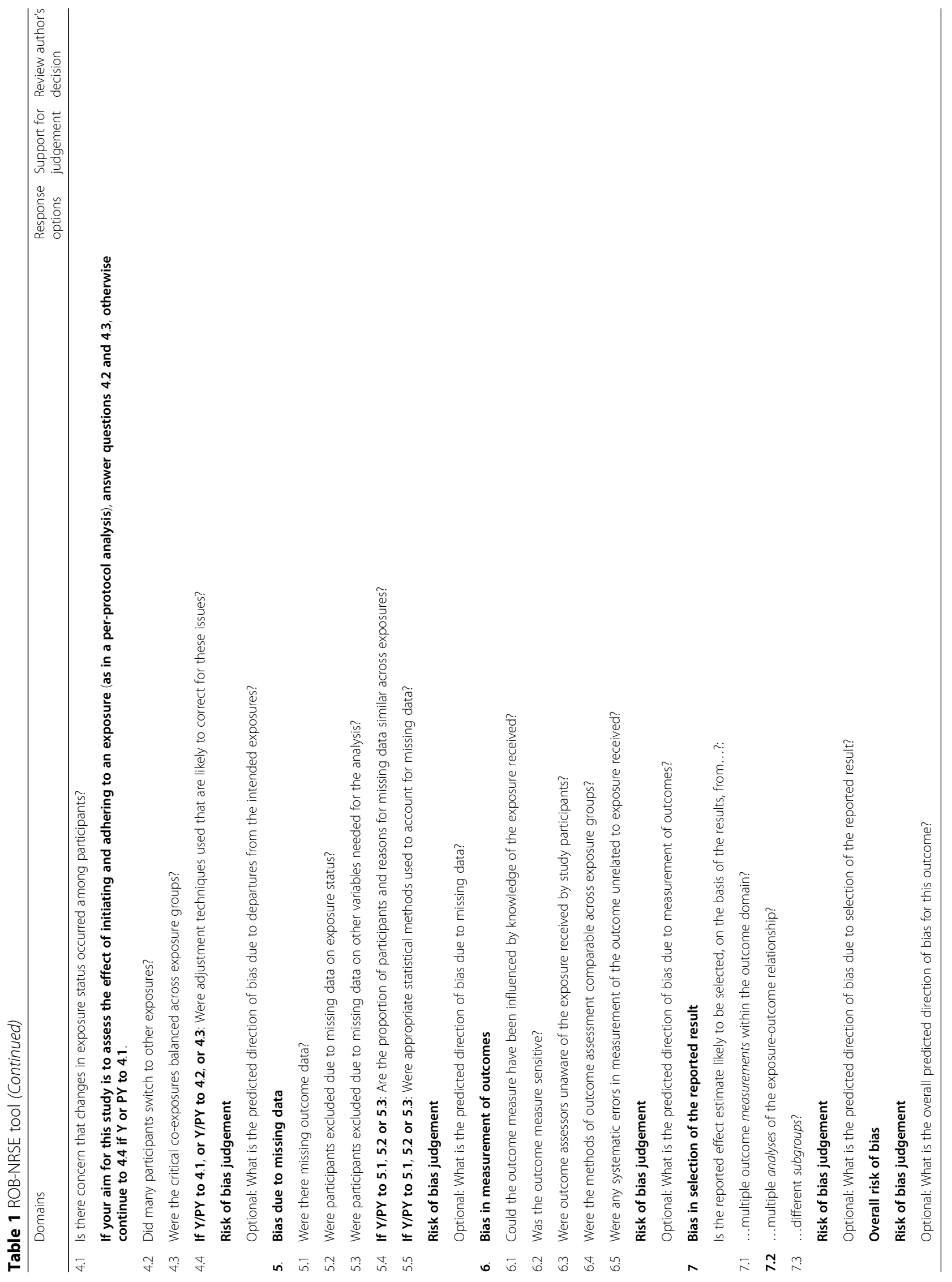


Table 2 Reporting of IRR and ICR for ROB-NRSE (with or without guidance)

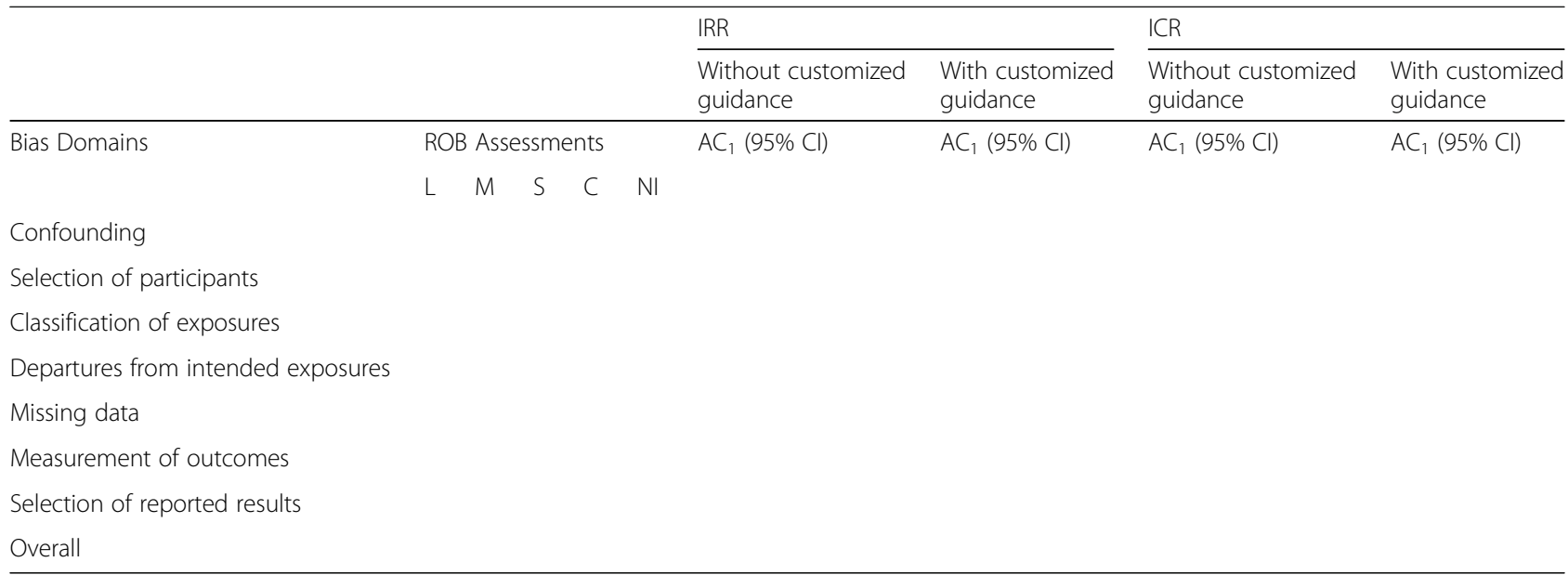

L low, $M$ moderate, $S$ serious, $C$ critical, $N I$ no information

assessments. For comparison of overall assessments between the two tools, we will use the following algorithm: 0-2 stars in NOS will be considered similar to "critical ROB" in ROB-NRSE, 3-5 stars in NOS will be considered as similar to "serious ROB" in ROB-NRSE, 6-8 stars in NOS will be considered as similar to "moderate ROB" in ROB-NRSE, and 9 stars in NOS will be considered as similar to "low ROB" in ROB-NRSE. In addition, for any discordance observed between domains or overall assessment, we will explore the possible reasons and attempt to provide explanations.

\section{Data analysis}

An experienced biostatistician (RR) from the collaborating center will conduct all the analyses in collaboration with the other members of the research team. We will transfer all collected data from Excel workbook to SAS (9.4), (SAS Institute Inc., Cary, NC, USA) for analysis.

We will use the following algorithm for comparison between similar items (partially or completely overlapping) in the two tools (NOS and ROB-NRSE):

1. For the "selection" domain in NOS. Assessments with 4 stars will be considered equivalent to "low ROB" adjudication in ROB-NRSE. Assessments with 3 stars will be considered equivalent to "moderate ROB" adjudication in ROB-NRSE. Assessments with 2 stars will be considered equivalent to "serious ROB" adjudication in ROB-NRSE, and assessments with 0 or 1 star will be considered equivalent to "critical ROB" adjudication in ROB-NRSE.

2. For the "comparability" domain in NOS. Assessments with 2 stars will be considered equivalent to "low ROB" adjudication in ROBNRSE. Assessments with 1 star will be considered equivalent to "moderate ROB" adjudication in ROB-
NRSE. Assessments with 0 star will be considered equivalent to "serious or critical ROB" adjudication in ROB-NRSE.

3. For the "outcome assessment" domain in NOS.

Assessments with 3 stars will be considered equivalent to "low ROB" adjudication in ROBNRSE. Assessments with 2 stars will be considered equivalent to "moderate ROB" adjudication in ROBNRSE. Assessments with 1 star will be considered equivalent to "serious ROB" adjudication in ROBNRSE, and assessments with 0 star will be considered equivalent to "critical ROB" adjudication in ROB-NRSE.

4. The NOS domains with "no description/no statement" assessments will be considered equivalent to the "no information" adjudication in ROB-NRSE.

For measuring concordance or discordance between various domains of NOS and ROB-NRSE (i.e., to assess concurrent validity of ROB-NRSE ), we will use "Kendall's tau," a rank correlation coefficient statistic [32], and its 95\% confidence intervals (for ordinal variables) for each domain and for the overall assessments.

\section{III: Evaluator burden}

The time taken to apply any newly developed instrument is an important factor to consider, as it may contribute to significant burden on the evaluator/reviewer. It is also important to assess factors that could reduce the application time. In this study, we will compare the time taken to apply ROB-NRSE (without and with guidance), time taken by the reviewer pairs to arrive at a consensus (without and with guidance), and the time taken to apply NOS for comparison with ROB-NRSE. 


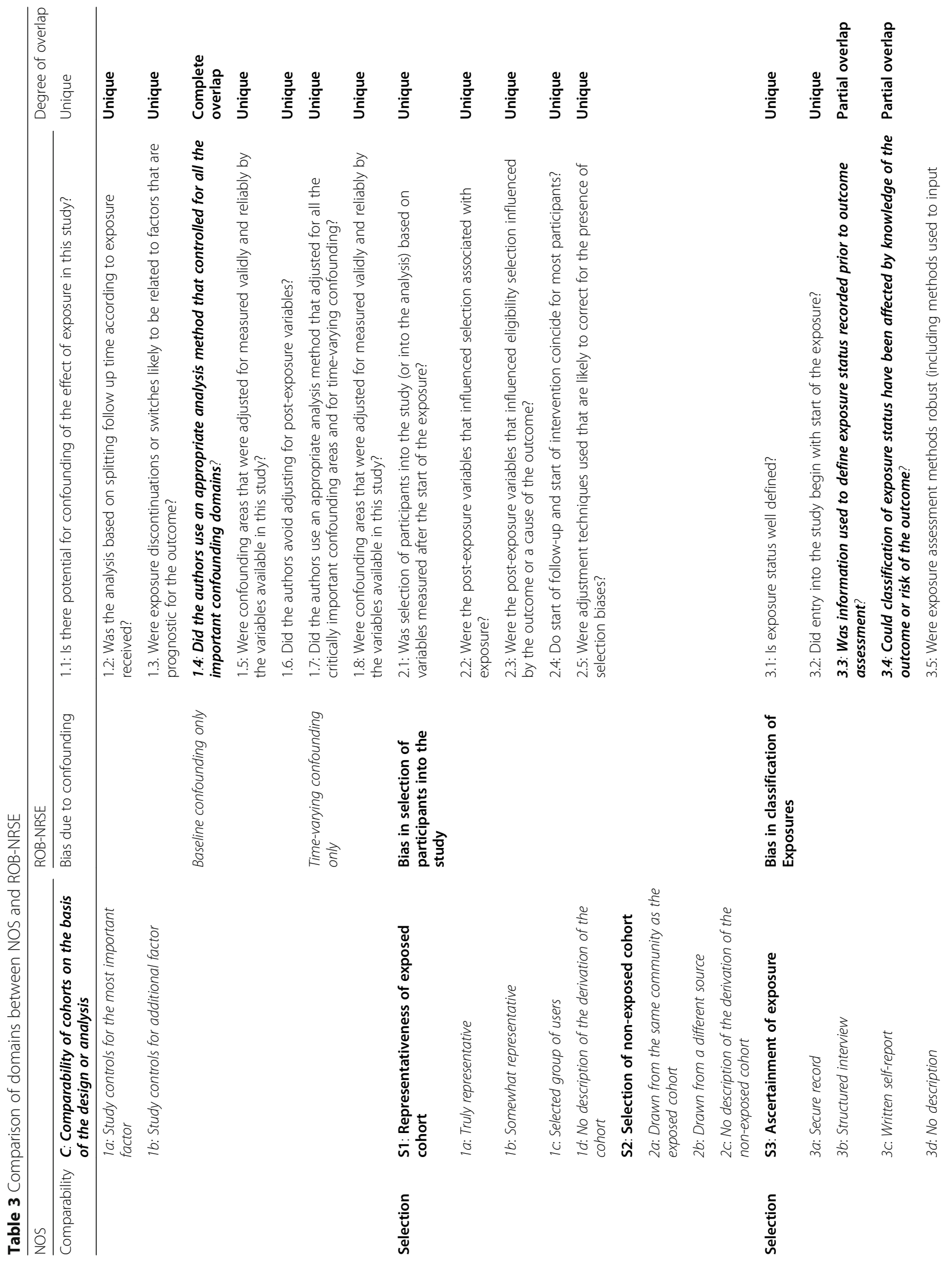




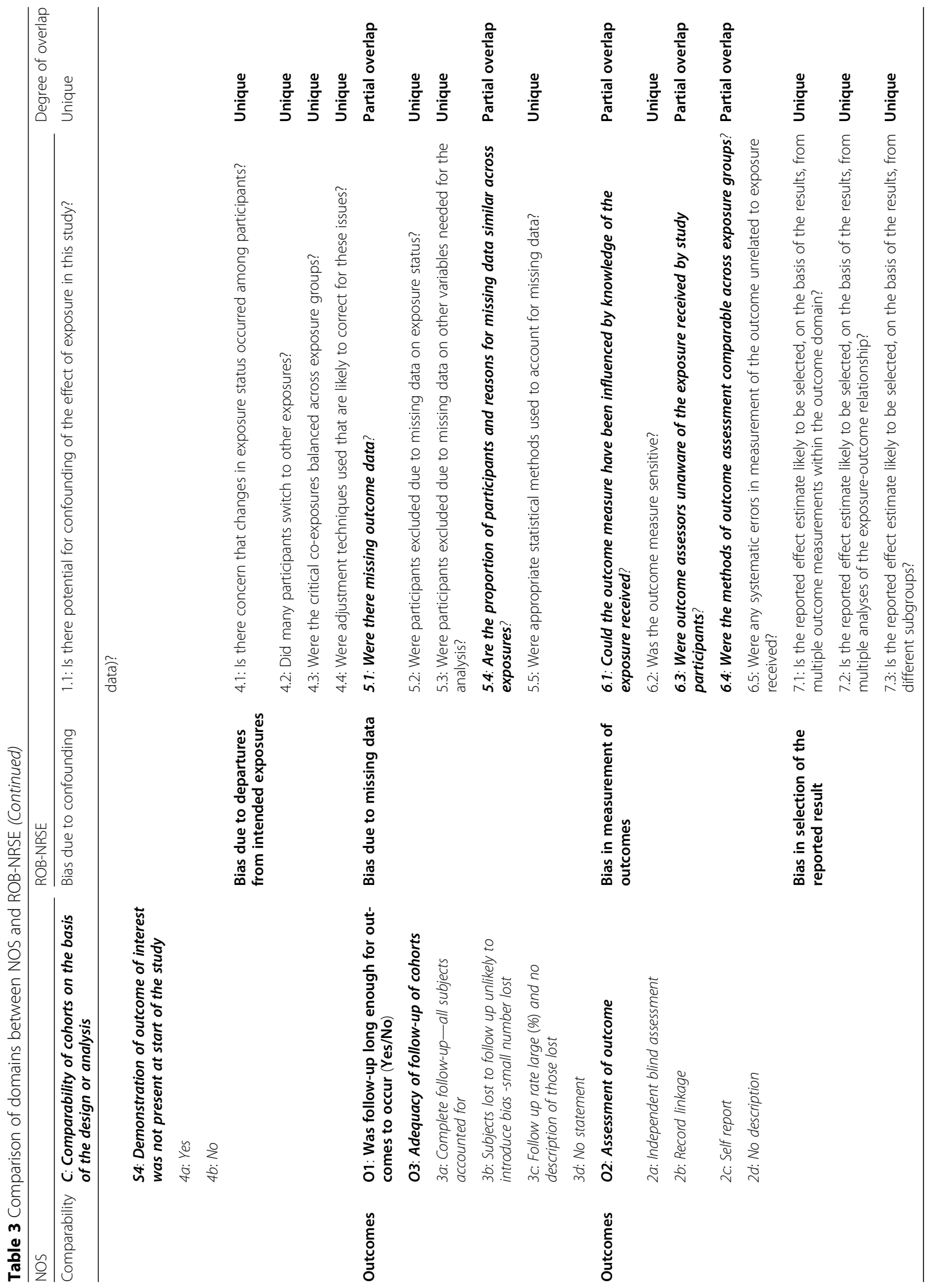


Table 4 Similar items between NOS [7] and ROB-NRSE

\begin{tabular}{|c|c|c|c|c|}
\hline & Similar Domains & $\begin{array}{l}\text { ROB-NRSE (signaling } \\
\text { questions) }\end{array}$ & $\begin{array}{l}\text { NOS } \\
\text { (domain items) }\end{array}$ & Degree of overlap \\
\hline 1. & $\begin{array}{l}\text { ROB-NRSE: Bias due to confounding } \\
\text { NOS: Comparability }\end{array}$ & 1.4 & C1a, C1b & Complete overlap \\
\hline 2. & $\begin{array}{l}\text { ROB-NRSE: Bias in selection of participants } \\
\text { NOS: Selection }\end{array}$ & - & - & Unique \\
\hline 3. & $\begin{array}{l}\text { ROB-NRSE: Bias in classification of exposures } \\
\text { NOS: Demonstration of outcome of interest was not present at start of the study }\end{array}$ & $3.3,3.4$ & S4a, S4b & Partial overlap \\
\hline 4. & $\begin{array}{l}\text { ROB-NRSE: Bias due to deviations from intended exposures } \\
\text { NOS: - }\end{array}$ & - & - & Unique \\
\hline 5. & $\begin{array}{l}\text { ROB-NRSE: Bias due to missing data } \\
\text { NOS: Adequacy of follow-up of cohorts }\end{array}$ & $5.1,5.4$ & O3a, O3b, O3c, O3d & Partial overlap \\
\hline 6. & $\begin{array}{l}\text { ROB-NRSE: Bias in measurement of outcomes } \\
\text { NOS: Assessment of outcome }\end{array}$ & $6.1,6.3$ & $\mathrm{O} 2 \mathrm{a}, \mathrm{O} 2 \mathrm{~b}, \mathrm{O} 2 \mathrm{c}, \mathrm{O} 2 \mathrm{~d}$ & Partial overlap \\
\hline 7. & $\begin{array}{l}\text { ROB-NRSE: Bias in selection of the reported result } \\
\text { NOS: - }\end{array}$ & - & - & Unique \\
\hline
\end{tabular}

\section{Data collection process}

Reviewers will record (using a digital clock) the time taken (in minutes) while applying (time to read article plus time to adjudicate) ROB-NRSE tool (without and with guidance), time taken for consensus, and the time taken to apply the NOS tool (time to read article plus time to adjudicate) for each included NRSE. The reviewers will use the Excel workbook created by the principal investigator to record the start time, end time, and total time to apply ROB-NRSE at the completion of the assessment for each NRSE and after the consensus process with the second reviewer. The reviewers will split the time to apply ROB-NRSE into the time taken to read the full text of the NRSE and the time taken for adjudications. The time to apply ROB-NRSE will begin when the reviewer begins reading the full texts of the NRSE and will end when decisions for all domains are completed and an overall ROB assessment for the study is established. The average overall time to apply ROB-NRSE for the same set of articles assessed by each reviewer will be calculated. In addition, we will also calculate the time taken to resolve conflicts and arrive at a consensus, and the overall time (time to apply plus time taken to arrive at a consensus) for each pair of reviewers. The time to arrive at a consensus will start when the two reviewers convene to resolve conflicts and will end when they arrive at a consensus.

\section{Data analysis}

An experienced biostatistician (RR) from the coordinating center will conduct all the analyses in collaboration with the other members of the research team. We will transfer all collected data from Excel workbook to SAS (9.4), (SAS Institute Inc., Cary, NC, USA) for analysis.
1. We will first summarize the average time (mean and SD) taken by the reviewers to assess ROB-

NRSE without guidance and with guidance separately.

2. To analyze the impact of customized training and guidance on changes in evaluator burden (ROBNRSE assessment time as well as the time taken by the reviewer pairs to arrive at consensus), we will compare two centers separately $(n=44$ respectively). We will use generalized linear models to evaluate changes in time taken to assess ROBNRSE after customized guidance (compared with without guidance). We will control for the correlation between reviewers using random effects. The distribution of outcome will be adjusted by using a link function.

3. To analyze the time taken to apply ROB-NRSE compared with NOS, we will use a fixed effect generalized linear model. The model distribution will be chosen by link function.

\section{Discussion}

Systematic reviews including NRSE can provide valuable evidence on rare outcomes, adverse events, longterm outcomes, real-world practice, and in situations where RCTs are not available [9, 33]. It is very important to appraise the ROB in the included NRSE to have a complete understanding of the strengths and weaknesses of the overall evidence, as methodological flaws in the design or conduct of the NRSE could lead to biased effect estimates [9]. The newly developed ROB-NRSE could be a very useful tool for researchers in assessing risk of bias in NRSE when undertaking systematic reviews of NRSE. As such, it is important to evaluate the usability, reliability, and concurrent validity of this tool to help identify 
potential barriers and facilitators in applying this tool in a real-world setting.

In this cross-sectional study protocol, we describe the methods we will use to assess the inter-rater reliability, inter-consensus reliability, and the concurrent validity of ROB-NRSE. Our proposed study, upon completion, will provide empirical evidence on the IRR, concurrent validity, and the evaluator burden of ROB-NRSE.

\section{Strengths and challenges}

Across the world, researchers, with a range of expertise, conduct systematic reviews that include NRSE. The ROB-NRSE tool was designed to be used by systematic reviewers with varied academic backgrounds and experience across multiple knowledge synthesis centers. A major strength of our study is that we will involve reviewers from multiple research teams with a range of expertise and academic backgrounds (highest degree attained) to apply and test ROB-NRSE, in order to simulate the real-world settings. We will also use a sample of NRSE that were not evaluated previously by the reviewers, in order to mimic what is typically encountered in a real-world setting. In addition, similar to what will be encountered in the real-world setting, we anticipate that the time taken to assess $\mathrm{ROB}$ might be longer for NRSE appraised at the beginning compared with those appraised later, due to increasing familiarity and a learning curve. To the best of our knowledge, there are no studies that have assessed the IRR, the ICR, the evaluator burden, the impact of additional training and guidance on IRR, ICR and the evaluator burden, and the construct validity of ROB-NRSE (comparison of ROB-NRSE with NOS). As with any elaborate tool, it is important to evaluate concerns regarding the practical use of ROBNRSE. The findings of our cross-sectional study have a potential to elucidate the impact of training and development of customized guidance with decision rules on the IRR, ICR, and the evaluator burden of ROB-NRSE. Also, for data analysis, we will use the $\mathrm{AC}_{1}$ statistic developed by Gwet [27] to calculate true chance agreement in the presence of high agreement between reviewers, thus yielding values closer to "true" IRR for ROB-NRSE.

We anticipate the following limitations. For feasibility, the reviewers will only appraise ROB for a single outcome for each NRSE. This may be a limitation as reviewers in real-world settings that may need to appraise multiple outcomes for each of the included NRSE and the evaluator burden might differ slightly from the findings of this study. In a real-world setting, the training and customized guidance decision rules developed by the researchers for their own systematic reviews may differ from the one developed by the principal investigator of this study, and this may pose a challenge in the generalization of the findings of this study. For feasibility, we have proposed to use the same reviewers for both stages (without and with guidance), and we anticipate that this may bias the effect of training and guidance. However, we will address this limitation by assessing the correlations between adjudications made during the two stages, for each of the reviewers. A poor correlation between adjudications made during the two stages for a reviewer would indicate that the training and guidance have been useful.

As with any new tool, it is critical to assess the IRR, ICR, concurrent validity, and evaluator burden of ROB-NRSE, in order to improve the consistency of its application and its interpretation across various systematic reviews that include NRSE. We hope that the findings of this study will contribute to an improved understanding and better application of the ROB instrument for NRS of exposures tool.

\section{Knowledge Dissemination strategy}

Systematic reviews serve as a source of knowledge and evidence to aid in the decision-making process. Our cross-sectional study addresses issues that may contribute to the quality of the evidence synthesized by the systematic review and thus will be of great interest to all stakeholders such as clinicians, decision-makers, patients, and the general-public through GRADE assessments of the quality of the evidence. It will also be of great interest to researchers conducting Cochrane and non-Cochrane systematic reviews to improve their understanding regarding the practical use of the ROBNRSE tool. We plan to disseminate the results of our cross-sectional study by presenting the study results at various conferences (e.g., Cochrane Colloquium) by publishing study results in academic journals and by spreading the message through social media (e.g., Twitter).

\section{Abbreviations \\ GRADE: Grading of Recommendations, Assessment, Development and Evaluation; ICR: Inter-consensus reliability; IRR: Inter-rater reliability; NLM: National Library of Medicine; NOS: Newcastle-Ottawa Scale; NRS: Non- randomized studies; NRSE: Non-randomized studies of exposures; NRSI: Non- randomized studies of interventions; RCT: Randomized controlled trials; ROB: Risk of bias; ROBINS-E: Risk of Bias in Non-randomized Studies of Expo- sures; ROBINS-I: Risk of Bias in Non-randomized Studies of Interventions; SD: Standard deviation; UK: United Kingdom; USA: United States of America; K: Kappa statistic}

\section{Acknowledgements}

Not applicable.

\section{Authors' contributions}

MJ and AMAS substantially contributed to the design and conception of the study. MJ drafted the protocol with input from all other team members. MJ, AMAS, and RR were involved in developing and writing the analysis plan in the protocol. MJ and MA will be involved in the development of the customized guidance with decision rules. LC, NA, RCR, CB, and KH will be involved in the ROB appraisals. All study authors read and approved the final protocol. 


\section{Funding}

There is no dedicated funding for this study. ACT is funded by a Tier 2 Canada Research Chair in Knowledge Synthesis.

\section{Availability of data and materials}

Not applicable.

\section{Ethics approval and consent to participate}

Not applicable.

\section{Consent for publication}

Not applicable.

\section{Competing interests}

The authors declare that they have no competing interests.

\section{Author details}

${ }^{1}$ The George \& Fay Yee Center for Healthcare Innovation, University of Manitoba, 363-753 McDermot Avenue, Winnipeg, Manitoba R3E 0 T6, Canada. ${ }^{2}$ Department of Community Health Sciences, University of Manitoba, Winnipeg, Canada. ${ }^{3}$ Li Ka Shing Knowledge Institute, St. Michael's Hospital, Unity Health Toronto, Toronto, Canada. ${ }^{4}$ Pharmalytics Group, Vancouver, Canada. ${ }^{5}$ Evidinno Outcomes Research Inc., Vancouver, British Columbia, Canada. ${ }^{6}$ School of Epidemiology and Public Health, Faculty of Medicine, University of Ottawa, Ottawa, Canada. ${ }^{7}$ Epidemiology Division, Dalla Lana School of Public Health \& Institute of Health, Management, and Policy Evaluation, University of Toronto, Toronto, Canada. ${ }^{8}$ Queen's Collaboration for Health Care Quality Joanna Briggs Institute Centre of Excellence, Queen's University, Kingston, Ontario, Canada.

Received: 14 January 2020 Accepted: 5 February 2020

Published online: 12 February 2020

\section{References}

1. Treadwell JR, Singh S, Talati R, McPheeters ML, Reston JT. A framework for "Best Evidence" approaches in systematic reviews. Rockville (MD)2011.

2. Schunemann HJ, Cuello C, Akl EA, et al. GRADE guidelines: 18. How ROBINSI and other tools to assess risk of bias in nonrandomized studies should be used to rate the certainty of a body of evidence. Journal of clinical epidemiology. 2018.

3. Norris S, Atkins D, Bruening W, et al. Selecting observational studies for comparing medical interventions. Rockville (MD): Methods Guide for Effectiveness and Comparative Effectiveness Reviews; 2008.

4. O'Neil M, Berkman N, Hartling L, et al. Observational evidence and strength of evidence domains: case examples. Systematic reviews. 2014;3:35.

5. Hartling L, Milne A, Hamm MP, et al. Testing the Newcastle Ottawa Scale showed low reliability between individual reviewers. Journal of clinical epidemiology. 2013;66(9):982-93.

6. Downs $\mathrm{SH}$, Black N. The feasibility of creating a checklist for the assessment of the methodological quality both of randomised and non-randomised studies of health care interventions. Journal of epidemiology and community health. 1998;52(6):377-84

7. Wells GA, Shea B, O'Connell Dea. The Newcastle-Ottawa Scale (NOS) for assessing the quality of nonrandomised studies in meta-analyses. http:// www.ohri.ca/programs/clinical_epidemiology/oxford.asp. 2008.

8. Sanderson S, Tatt ID, Higgins JP. Tools for assessing quality and susceptibility to bias in observational studies in epidemiology: a systematic review and annotated bibliography. International journal of epidemiology. 2007;36(3):666-76.

9. Sterne JA, Hernan MA, Reeves BC, et al. ROBINS-l: a tool for assessing risk of bias in non-randomised studies of interventions. Bmj. 2016;355:14919.

10. Morgan RL, Thayer KA, Santesso N, et al. Evaluation of the risk of bias in non-randomized studies of interventions (ROBINS-I) and the 'target experiment' concept in studies of exposures: rationale and preliminary instrument development. Environ Int. 2018;120:382-7.

11. Higgins JP, Altman DG, Gotzsche PC, et al. The Cochrane Collaboration's tool for assessing risk of bias in randomised trials. Bmj. 2011;343:d5928,

12. Whiting P, Savovic J, Higgins JP, et al. ROBIS: a new tool to assess risk of bias in systematic reviews was developed. Journal of clinical epidemiology. 2016;69:225-34
13. Khan A, Boukrina O, Oh-Park M, Flanagan NA, Singh M, Oldham M. Preventing delirium takes a village: systematic review and meta-analysis of delirium preventive models of care. Journal of hospital medicine. 2019;14:E1-7.

14. Morgan RL, Thayer K, Santesso N, et al. Reliability and validity assessment of a risk-of-bias instrument for non-randomised studies of exposures. https:// abstracts.cochrane.org/2017-global-evidence-summit/reliability-and-validityassessment-risk-bias-instrument-non-randomised. 2017.

15. Morgan RL, Thayer KA, Santesso N, et al. A risk of bias instrument for nonrandomized studies of exposures: a users' guide to its application in the context of GRADE. Environ Int. 2019;122:168-84.

16. Cook DA, Beckman TJ. Current concepts in validity and reliability for psychometric instruments: theory and application. The American journal of medicine. 2006;119(2):166 e167-116.

17. Downing SM. Validity: on meaningful interpretation of assessment data. Medical education. 2003:37(9):830-7.

18. Jeyaraman MM, Rabbani R, Al-Yousif N, et al. Inter-rater reliability and concurrent validity of ROBINS-I: protocol for a cross-sectional study. Systematic reviews. 2020; [In-Press].

19. Mueller M, D'Addario M, Egger M, et al. Methods to systematically review and meta-analyse observational studies: a systematic scoping review of recommendations. BMC medical research methodology. 2018;18(1):44.

20. Vandenbroucke JP, von Elm E, Altman DG, et al. Strengthening the reporting of observational studies in epidemiology (STROBE): explanation and elaboration. Annals of internal medicine. 2007;147(8):W163-94.

21. von Elm E, Altman DG, Egger M, et al. Strengthening the reporting of observational studies in epidemiology (STROBE) statement: guidelines for reporting observational studies. Bmj. 2007;335(7624):806-8.

22. Kim JS, Phan K, Cheung ZB, et al. Surgical, radiographic, and patient-related risk factors for proximal junctional kyphosis: a meta-analysis. Global spine journal. 2019;9(1):32-40.

23. Cantor AB. Sample-size calculations for Cohen's kappa. Psychological methods. 1996;1(2):150-3.

24. Gwet KL. Sample-size determination. Inter-rater reliability discussion corner. http://agreestat.com/blog_irr/sample_size_determination.html. Last accessed on July 23, 2018. 2010.

25. Banzi R, Cinquini M, Gonzalez-Lorenzo M, Pecoraro V, Capobussi M, Minozzi S. Quality assessment versus risk of bias in systematic reviews: AMSTAR and ROBIS had similar reliability but differed in their construct and applicability. Journal of clinical epidemiology. 2018.

26. Cohen J. Weighted kappa: nominal scale agreement with provision for scaled disagreement or partial credit. Psychological bulletin. 1968;70(4):213-20.

27. Gwet KL. Computing inter-rater reliability and its variance in the presence of high agreement. The British journal of mathematical and statistical psychology. 2008;61(Pt 1):29-48.

28. Wongpakaran N, Wongpakaran T, Wedding D, Gwet KL. A comparison of Cohen's kappa and Gwet's AC1 when calculating inter-rater reliability coefficients: a study conducted with personality disorder samples. BMC Med Res Methodol. 2013;13:61.

29. Landis JR, Koch GG. The measurement of observer agreement for categorical data. Biometrics. 1977;33(1):159-74.

30. DeVon HA, Block ME, Moyle-Wright P, et al. A psychometric toolbox for testing validity and reliability. J Nurs Scholarship. 2007;39(2):155-64.

31. Higgins JPT, Green S, (editors). Cochrane handbook for systematic reviews of interventions version 5.1.0 [updated March 2011]. The Cochrane Collaboration, 2011. Available from www.handbook.cochrane.org.

32. Kendall MG. A new measure of rank correlation. Biometrika. 1938;30(1-2):81-93.

33. Hernan MA, Robins JM. Using big data to emulate a target trial when a randomized trial is not available. American journal of epidemiology. 2016; 183(8):758-64.

\section{Publisher's Note}

Springer Nature remains neutral with regard to jurisdictional claims in published maps and institutional affiliations. 